\title{
A Single-institution Study on Predictors of Short-term Progression from Mild Cognitive Impairment in Parkinson's Disease to Parkinson's Disease with Dementia
}

\author{
Yuki Tajiri," Kenji Wada-Isoe, ${ }^{*} \dagger$ Kenichiro Tanaka," Tadashi Adachi,* Ritsuko Hanajima* and Kenji Nakashima: \\ *Division of Neurology, Department of Brain and Neurosciences, Tottori University Faculty of Medicine, Yonago 683-8504, Japan, \\ †Department of Dementia Research, Kawasaki Medical School, Okayama 700-8505, Japan, and \$National Hospital Organization \\ Matsue Medical Center, Matsue 690-8556, Japan
}

\begin{abstract}
Background Patients with non-demented Parkinson's disease (PD) sometime have mild cognitive impairment (MCI), and mild cognitive impairment in Parkinson's disease (PD-MCI) may convert to Parkinson's disease with dementia (PDD) within several years. Cognitive impairment also occurs in the early stages of the disease, gradually progressing to lower quality of life and instrumental activities of daily living. It is important to elucidate the predictors of progression from PD-MCI to PDD via longitudinal studies.
\end{abstract}

Methods This was a single center, case-control study. We analysed data from 49 patients with PD-MCI diagnosed as level I using the Movement Disorder Society PD-MCI criteria at baseline who had completed 1.5 years of follow-up. We defined patients who progressed to PDD as patients with progressive PD-MCI and patients who did not progress to PDD as patients with non-progressive PD-MCI. Depression, apathy, sleep disorders, constipation, light-headedness, hallucinations, impulse control disorders (ICDs) and impulsive-compulsive behaviors (ICBs) at baseline were statistically analysed as predictors of progression.

Results Of the 49 PD-MCI patients, 33 did not convert to PDD (non-progressive PD-MCI), and 16 converted to PDD (progressive PD-MCI). The Mini-Mental State Examination (MMSE) score, light-headedness and ICDs were elucidated as predictors of progressive PD-MCI via a multivariate logistic regression model. The adjusted odds ratios (ORs) and 95\% confidence intervals (CIs) for each item were MMSE score, OR $0.324,95 \%$ CI $0.119-0.882, P=0.027$; light-headedness, OR 27.665, 95\% CI 2.263-338.185, $P=0.009$; and ICDs, OR 53.451, 95\% CI 2.298-291.085, $P=0.010$.

Conclusion Cognitive function, ICDs and lightheadedness may be risk factors for the development of PDD in PD-MCI patients.

Key words cognition; dementia; impulse control disorders; light-headedness; Parkinson's disease
Patients with Parkinson's disease (PD) have progressive motor symptoms such as muscle rigidity, tremor, loss of postural reflex, akinesia and bradykinesia caused by neurodegeneration of dopaminergic neurons of the substantia nigra and striatum. The definition of the disease was based on these motor symptoms, ${ }^{1}$ but it has been found that various non-motor symptoms, such as autonomic neuropathy (constipation, orthostatic hypotension, dysuria, sweating disorder and others), psychiatric symptoms (depression, apathy and hallucinations), sleep disorders, behavioral abnormalities [impulse control disorders (ICDs)] and cognitive impairment, can be complicated. The cognitive impairment of PD is thought to be due to cerebrovascular pathology, Alzheimer's disease pathology, Lewy body pathology that characterizes pathology of PD and a number of other mechanisms. ${ }^{2}$ In recent years, Lewy body pathology based on the Braak hypothesis is considered to be the main pathology of cognitive impairment of PD. ${ }^{2}$ Braak proposed that Lewy body development would first ascend the brainstem to the midbrain and substantia nigra and eventually damage the cerebral cortex. ${ }^{3,4}$ This hypothesis is broadly accepted. The Meynert basal ganglia, amygdala, and cerebral cortex are thought to be the focus of cognitive impairment of PD, but there are still many unclear cases. A disease concept of Parkinson's disease with dementia (PDD) has been proposed and investigated. A

Corresponding author: Yuki Tajiri, MD

y.tajiri.131@gmail.com

Received 2019 November 21

Accepted 2019 December 20

Online published 2020 January 9

Abbreviations: CI, confidence interval; DDS, dopamine dysregulation syndrome; ESS, Epworth Sleepiness Scale; GDS, Geriatric Depression Scale; ICBs, impulsive-compulsive behaviors; ICDs, impulse control disorders; LED, total L-dopa equivalent dose; MCI, mild cognitive impairment; MDS, Movement Disorder Society; MMSE, Mini-Mental State Examination; MoCA-J, the Japanese version of the Montreal Cognitive Assessment; OR, odds ratio; PD, Parkinson's disease; PDD, Parkinson's disease with dementia; PD-MCI, mild cognitive impairment in Parkinson's disease; PSQI, Pittsburgh Sleep Quality Index; QUIP, Questionnaire for Impulsive-Compulsive Disorders; RBD, rapid eye movement sleep behavior disorder; RBDSQ-J, RBD screening questionnaire Japanese version; REM, rapid eye movement; UPDRS-III, motor part of the Unified Parkinson's Disease Rating Scale 
systematic review reports the point prevalence of PDD is about $30 \% .^{5}$ There is also a report that the prevalence of dementia rate is $83 \%$ at 20 years after the onset of PD. ${ }^{6}$ Initially, it was believed that cognitive impairment occurred during the advanced stages of PD. ${ }^{7}$ However, it has been found that many patients have cognitive impairment from the first diagnosis of $\mathrm{PD}$. The concept of mild cognitive impairment in PD (PD-MCI) has been proposed as a prodromal stage of PDD. However, each researcher had different definitions of PD-MCI. ${ }^{8}$ Recently, a clinical diagnostic criteria for PD-MCI has been classified. ${ }^{9}$ PD-MCI is evident in $10-20 \%$ patients at the time of PD diagnosis. ${ }^{6}$ Among PD patients without dementia approximately $25-30 \%$ are PD-MCI. ${ }^{6}$ PD-MCI is suggested to be an identical risk factor for developing to PDD. ${ }^{10}$ However, the predictive factors for the progression from PD-MCI to PDD have not been well analysed. In this study, to elucidate the predictors for progression from PD-MCI to PDD, we compared the motor and non-motor symptoms between progression and non-progression groups of PD-MCI to PDD.

\section{MATERIALS AND METHODS}

We conducted a case-control study within a cohort to investigate the predictors of progression. The observation period was from 2012 to 2016.

\section{Patient Data}

Patients with PD who were treated at Tottori University Hospital were included in this study. At baseline, PDD and normal cognitive patients were excluded.

We performed the Mini-Mental State Examination (MMSE) and Japanese version of the Montreal Cognitive Assessment (MoCA-J) for neuropsychological evaluation. At the initial diagnosis of PD-MCI, in addition to neuropsychological examinations, we evaluated the motor part of the Unified Parkinson's Disease Rating Scale (UPDRS-III) as motor symptoms and asked the patients themselves and their caregivers or family members to respond to awareness of the nonmotor symptoms by filling in questionnaires. We chose the following as items of non-motor symptoms: depression by the Geriatric Depression Scale (GDS) and the Apathy Scale, ${ }^{11}$ sleep abnormalities by the Pittsburgh Sleep Quality Index (PSQI) and the Epworth Sleepiness Scale (ESS), ${ }^{12}$ rapid eye movement sleep behavior disorder (RBD) by the RBD screening questionnaire [Japanese version] (RBDSQ-J), ${ }^{13}$ awareness of constipation, awareness of head rush (light-headedness) as orthostatic hypotension, awareness of hallucinations, impulse control disorders (ICDs) [pathological gambling (gambling), excessive sexuality (hypersexuality), compulsive shopping (shopping) and binge eating (eating)] and impulsive-compulsive behaviors (ICBs) [hobbyism, repetition of the same simple behavior (punding) and excessive craving for dopamine drug use (dopamine dysregulation syndrome: DDS)] by the Questionnaire for Impulsive-Compulsive Disorders (QUIP). ${ }^{14}$ In QUIP, one or two questions are prepared for each ICDs and ICBs item, and if one is checked, the diagnosis is made.

Demographic information included sex, age at disease onset, age at first examination, disease duration, years of education and total L-dopa equivalent doses (total LEDs).

The diagnosis of PD was made according to the diagnostic criteria of the UK Brain Bank. ${ }^{15}$ Patients with PDD were identified according to the Movement Disorder Society (MDS) diagnostic criteria of 2007. ${ }^{16,17}$ Among non-PDD patients, patients with PD-MCI were identified according to the MDS criteria of 2012.9, 17 PD-MCI level I criteria was applied for this research. ${ }^{18}$

We identified patients with PD-MCI among patients with PD. After an observation period of 1.5 years, we again classified their cognitive diagnosis according to the same diagnostic criteria.

When the PD-MCI group was observed over time, a group proceeding to PDD (PD-MCI converter), a group in the state of PD-MCI (PD-MCI stable) and a group returning to normal cognition (PD-MCI reverter) were identified. We defined the PD-MCI converter group as progressive PD-MCI, and defined PD-MCI stable group and the PD-MCI reverter group as nonprogressive PD-MCI (Fig. 1).

\section{Statistical Analysis}

The relative risk of progression from PD-MCI to PDD was estimated as odds ratio (OR) using logistic regression models. First, the difference between the two groups was analyzed via Student's $t$-test for normally distributed variables, the Mann-Whitney $U$ test for nonparametric data and the chi-square test for comparison of frequencies. Next, variables that differed between the two groups at $P<0.1$ by bivariate analysis were selected. Pearson's and Spearman's correlation coefficients between the independent variables of the selected items were calculated and judged whether or not they were suitable candidates for logistic regression analysis. Selected items were incorporated in the logistic regression models. ORs were calculated in bivariate regression models and a multivariate regression model. In a multivariate logistic regression model, suitable predictor variables were selected using a backward likelihood ratio test to avoid arbitrary judgment. The fitness of these models was evaluated using the Hosmer-Lemeshow method. $P$ values less than 0.05 were considered to 


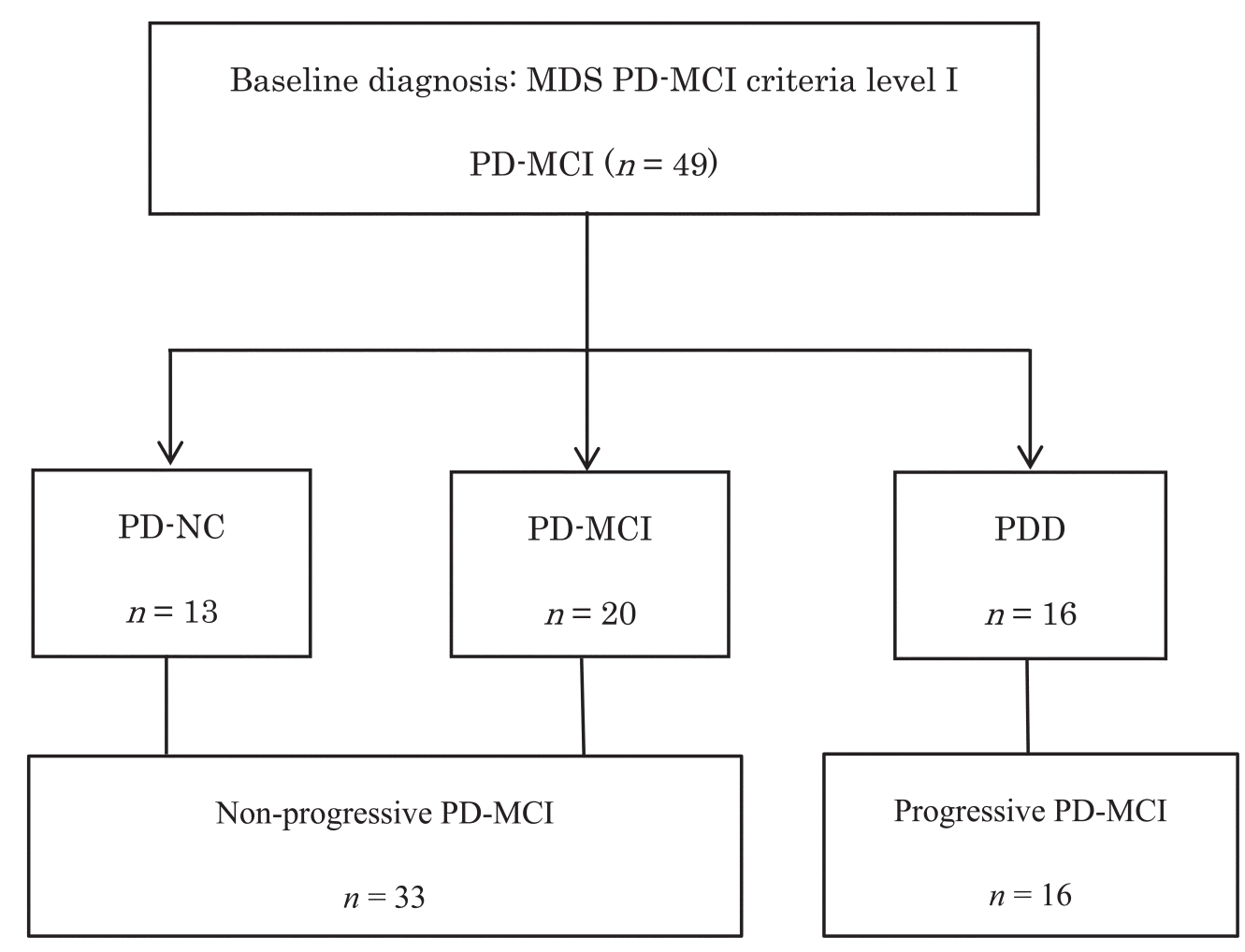

Fig. 1. Cognitive progression of PD-MCI. The number of PD-MCI patients at baseline $(n=49)$ and the numbers of PD-NC, PD-MCI and PDD patients after 1.5 years. The Movement Disorder Society (MDS) diagnostic criteria were used to diagnose cognitive function status. PD-NC, PD patients with normal cognition.

indicate statistical significance. Data analysis was conducted using IBM SPSS for Windows, version 22.

\section{Ethical Approval}

The study was conducted in accordance with the current revision of the Declaration of Helsinki and applicable national laws and regulations. It was approved by the Medical Ethics Committee of Tottori University (approval number 2178).

\section{RESULTS}

We identified 49 patients diagnosed with PD-MCI during the observation period for whom follow-up surveillance could be conducted. Of the 49 PD-MCI patients, 16 patients converted to PDD (progressive PD-MCI), 20 patients remained in PD-MCI (stable PD-MCI) and 13 patients reverted to normal cognitive level (reverted PD-MCI). We defined the reverted and stable PD-MCI groups (33 patients) as the non-progressive PD-MCI group (Fig. 1). No patients used anticholinergic drugs or anti-dementia drugs (donepezil, rivastigmine, galantamine or memantine) during the observation period.

There were no missing data for sex, age at disease onset, age at first examination, disease duration, years of education, MoCA-J, MMSE, GDS, Apathy Scale,
RBDSQ-J, constipation, light-headedness, hallucinations, ICDs and ICBs. Data were missing for 1 patient for total LEDs, 24 patients for UPDRS-III score and 2 patients for PSQI and ESS.

In bivariate analysis, MMSE, ESS, light-headedness, ICDs, gambling, hypersexuality, shopping and DDS were selected at $P<0.1$ (Table 1). The absolute values of the Pearson and the Spearman correlation coefficient between the independent variables of the selected items were less than 0.7 , and all items were incorporated as candidates for the logistic regression model. Using these eight items, ORs were calculated using bivariate logistic models and multivariate logistic models.

The bivariate logistic models showed that lightheadedness, gambling, hypersexuality and ICDs were statistically significant as predictors of progressive PD-MCI at $P<0.05$ (Table 2). The OR for lightheadedness was 4.776 (95\% CI 1.310-17.402), the OR for gambling was 7.045 (95\% CI 1.190-41.705), the OR for hypersexuality was 14.545 (95\% CI $1.527-138.511)$ and the OR for ICDs was 4.500 (95\% CI 1.202-16.848).

The multivariate logistic regression models showed that MMSE, light-headedness and ICDs were selected as variables of the model. Age, sex and years of 
Table 1. Clinical Characteristics of progressive and non-progressive PD-MCI patients

\begin{tabular}{|c|c|c|c|c|c|}
\hline \multirow{2}{*}{\multicolumn{2}{|c|}{ Characteristic }} & Overall & $\begin{array}{l}\text { Non-progressive } \\
\text { PD-MCI }\end{array}$ & $\begin{array}{l}\text { Progressive } \\
\text { PD-MCI }\end{array}$ & \multirow{2}{*}{$\begin{array}{c}P \text {-value } \\
* P<0.1 \\
* * P<0.05\end{array}$} \\
\hline & & $n=49$ & $n=33$ & $n=16$ & \\
\hline Sex (male: female) & & $24: 25$ & $15: 18$ & $9: 7$ & 0.478 \\
\hline Age at disease onset (years) & mean (SD) & $60.63(11.0)$ & $60.42(11.1)$ & $61.06(11.3)$ & 0.852 \\
\hline Age at first examination (years) & mean (SD) & $70.31(7.9)$ & $70.03(7.8)$ & $70.88(8.2)$ & 0.729 \\
\hline Disease duration (years) & mean (SD) & $9.20(7.6)$ & $9.12(8.1)$ & $9.38(6.6)$ & 0.914 \\
\hline Education (years) & mean (SD) & $12.49(2.8)$ & $12.55(3.1)$ & $12.3(2.3)$ & 0.845 \\
\hline Total LEDs (mg) & mean (SD) & $467.4(319.9)$ & $420.8(284.2)$ & $570.1(377.5)$ & 0.135 \\
\hline UPDRS-III score (points) & median (IQR) & $21.96(17.00-27.50)$ & $21.05(15.00-27.75)$ & $25.60(22.00-29.50)$ & 0.394 \\
\hline MMSE (points) & median (IQR) & $27.57(26.00-29.00)$ & $27.85(27.00-29.00)$ & $27.00(26.00-28.00)$ & $0.073 *$ \\
\hline GDS (points) & median (IQR) & $5.41(2.00-7.50)$ & $4.97(1.00-8.00)$ & $6.31(4.00-7.75)$ & 0.151 \\
\hline Apathy Scale (points) & median (IQR) & $15.73(11.50-19.00)$ & $15.33(11.50-19.50)$ & $16.56(11.00-19.00)$ & 0.528 \\
\hline PSQI (points) & median (IQR) & $6.32(3.00-9.00)$ & $5.91(3.00-8.75)$ & $7.20(4.00-10.00)$ & 0.281 \\
\hline ESS (points) & median (IQR) & $7.04(4.00-10.00)$ & $6.34(3.00-9.75)$ & $8.53(4.00-12.00)$ & $0.075 *$ \\
\hline RBDSQ (points) & median (IQR) & $4.29(1.50-6.00)$ & $3.82(1.00-5.50)$ & $5.25(2.00-7.75)$ & 0.127 \\
\hline Constipation & $n(\%)$ & $38(77.6 \%)$ & $24(72.7 \%)$ & $14(87.5 \%)$ & 0.216 \\
\hline Light- headedness & $n(\%)$ & $16(32.7 \%)$ & $7(21.2 \%)$ & $9(56.3 \%)$ & $0.014 * *$ \\
\hline Hallucinations & $n(\%)$ & $9(18.4 \%)$ & $5(15.2 \%)$ & $4(25.0 \%)$ & 0.322 \\
\hline ICD \& ICB & $n(\%)$ & $21(42.9 \%)$ & $12(36.4 \%)$ & $9(56.3 \%)$ & 0.187 \\
\hline ICD & $n(\%)$ & $13(26.5 \%)$ & $5(15.2 \%)$ & $8(50.0 \%)$ & $0.014 * *$ \\
\hline Gambling & $n(\%)$ & $7(14.3 \%)$ & $2(6.1 \%)$ & $5(31.3 \%)$ & $0.030 * *$ \\
\hline Hypersexuality & $n(\%)$ & $6(12.2 \%)$ & $1(3.0 \%)$ & $5(31.3 \%)$ & $0.011 * *$ \\
\hline Shopping & $n(\%)$ & $4(8.2 \%)$ & $1(3.0 \%)$ & $3(18.8 \%)$ & $0.096^{*}$ \\
\hline Eating & $n(\%)$ & $7(14.3 \%)$ & $3(9.1 \%)$ & $4(25.0 \%)$ & 0.146 \\
\hline ICB & $n(\%)$ & $12(25.0 \%)$ & $8(28.1 \%)$ & $4(25.0 \%)$ & 0.608 \\
\hline Hobbyism & $n(\%)$ & $6(12.2 \%)$ & $3(9.1 \%)$ & $3(18.8 \%)$ & 0.298 \\
\hline Punding & $n(\%)$ & $6(12.2 \%)$ & $4(12.1 \%)$ & $2(12.5 \%)$ & 0.649 \\
\hline DDS & $n(\%)$ & $6(12.2 \%)$ & $2(6.1 \%)$ & $4(25.0 \%)$ & $0.080^{*}$ \\
\hline
\end{tabular}

Statistical analysis was performed as follows: chi-square test for sex, constipation, light-headedness, hallucinations, gambling, hypersexuality, shopping, eating, hobbyism, punding and DDS. T-test for age at onset, age at examination, disease duration, education and total LEDs. Mann-Whitney $U$ test for USDRS-IIIscore, MMSE, GDS, Apathy Scale, PSQI, ESS and RBDSQ. IQR, Interquartile range.

education were also adjusted. The Hosmer-Lemeshow chi-square value was 0.338 , suggesting a good fit of the model. The discriminant predictive value was $75.6 \%$, and the model was effective for prediction. There were no outliers with predicted values exceeding 3 SD. In the multivariate logistic regression model, the adjusted ORs for each item were as follows: MMSE score (OR 0.324, 95\% CI 0.119-0.882, $P=0.027$ ), light-headedness (OR $27.665,95 \%$ CI 2.263-338.185, $P=0.009$ ) and ICDs (OR $53.431,95 \%$ CI 2.298-291.085, $P=0.010$ ).

\section{DISCUSSION}

The present study identified not only the MMSE score of the cognitive scale, but also light-headedness and
ICDs as risk factors for progression from PD-MCI to PDD by using multiple logistic regression analysis. Although, many studies regarding the risk for progression from non-PDD to PDD have been reported, a few studies have reported the risk for progression from PD-MCI to PDD. In previous papers, cognitive functions such as visuospatial, semantic language, episodic memory and mental flexibility were reported to relate with the progression of PDD. ${ }^{19,20}$ To our knowledge, this is the first report that light-headedness and ICDs are risk factors for converting from PD-MCI to PDD.

\section{Light-headedness}

Autonomic nervous system disorders cause orthostatic 
Table 2. Relative risk of progression from PD-MCI to PDD

\begin{tabular}{|c|c|c|c|c|c|c|}
\hline & \multicolumn{3}{|c|}{ Bivariate odds ratios } & \multicolumn{3}{|c|}{ Multivariate-adjusted odds ratios } \\
\hline & ORs & $95 \% \mathrm{CI}$ & $P$ & ORs & $95 \% \mathrm{CI}$ & $P$ \\
\hline MMSE & 0.651 & $0.412-1.028$ & 0.065 & 0.324 & $0.119-0.882$ & 0.027 \\
\hline ESS & 1.096 & $0.965-1.245$ & 0.159 & - & - & - \\
\hline Light-headedness & 4.776 & $1.310-17.402$ & 0.018 & 27.665 & $2.263-338.185$ & 0.009 \\
\hline Gambling & 7.045 & $1.190-41.705$ & 0.031 & - & - & - \\
\hline Hypersexuality & 14.545 & $1.527-138.511$ & 0.020 & - & - & - \\
\hline Shopping & 7.385 & $0.702-77.679$ & 0.096 & - & - & - \\
\hline DDS & 5.167 & $0.834-32.000$ & 0.078 & - & - & - \\
\hline ICDs & 4.500 & $1.202-16.848$ & 0.026 & 53.451 & $2.298-291.085$ & 0.010 \\
\hline
\end{tabular}

Bivariate odds ratios were calculated using bivariate logistic regression models. Multivariate odds ratios were calculated using multivariate logistic regression models with adjustment for age, sex and years of education. To identify suitable predictable variables, predictable variables were selected using a backward likelihood ratio test.

hypotension in PD patients. When orthostatic hypotension develops, the patient feels light-headedness as a subjective symptom. Orthostatic hypotension has been reported to be a risk factor for cognitive dysfunction in PD (non-PDD to PDD), ${ }^{21,22}$ but there has been no report of light-headedness as a risk factor. Our results may support the hypothesis that orthostatic hypotension is a risk factor for progression of cognitive impairment in PD. However, our study did not evaluate the patients' blood pressure and did not accurately refer to the relationship between light-headedness and orthostatic hypotension. It is necessary to study the relationship between light-headedness and orthostatic hypotension. Light-headedness may be related to factors other than orthostatic hypotension, including brain blood flow insufficiency due to arterial sclerosis such as the basilar artery or carotid artery. Light-headedness may be a useful question in clinical practice because the risk of progression can be easily confirmed without tests of orthostatic hypotension only by asking the PD-MCI patient whether light-headedness exists or not.

\section{ICDs}

The association between ICDs and cognitive function in Parkinson's disease is complex. A meta-analysis study has shown that patients with ICDs have impaired setshifting and reward-related decision-making abilities. ${ }^{23}$ There is another study that has compared cognitive domains according to with or without ICDs in patients with PD-NC, PD-MCI and PDD. In this study, there was no significant difference in the cognitive function of the PD-NC with or without ICDs group; however, the PD-MCI with ICDs group had impaired attention domain than the PD-MCI without ICDs group. Furthermore, the PDD with ICDs group showed poorer performance in the executive domain than the PDD without ICDs group. ${ }^{24}$ In addition, this study reported that in patients with PD-MCI as well as PDD patients with ICDs, frontal-striatal dysfunction (i.e., executive and attentive) may be more severe than posterior dysfunction (i.e., language and visuospatial). ${ }^{24}$ However, these reports about the relationship between PD-MCI and ICDs are all small cross-sectional studies with different results from study to study. There are still many unknowns. The present study is a valuable report that ICDs are associated with longitudinal PD-MCI cognitive progression. PD-MCI with ICDs have frontal dysfunction and may be suggesting pathological progression in the cerebral cortex and may be more likely to progress to PDD. It seems to fit Braak's model of development of Parkinson's disease pathology. ${ }^{3,4}$ Further studies, including longitudinal morphological and functional imaging and biological markers, may be necessary to clarify the question.

One limitation of this research is that it was a small and single center cohort study because there were few patients with PD-MCI whose cognitive function could be assessed longitudinally. In the future, longitudinal studies should be performed with more PD-MCI patients for more generalised information. Another limitation is that the PD-MCI diagnostic criteria were the level I criteria. Level II criteria ${ }^{9}$ might reveal more detailed features of the cognitive function of patients with progressive PD-MCI. The final limitation is the lack of drug information on amantadine that may affect cognitive function, and dopamine agonists that can be a risk for ICDs. These drug data should be reflected in future studies.

In conclusion, if a patient with PD-MCI has ICDs and light-headedness, then there is a risk of progression to PDD, and these symptoms should be considered in clinical practice. 
The authors declare no conflict of interest.

\section{REFERENCES}

1 Parkinson J. An essay on the shaking palsy. 1817. J Neuropsychiatry Clin Neurosci. 2002;14:223-36. DOI: 10.1176/ jnp.14.2.223, PMID: 11983801

2 Halliday GM, Leverenz JB, Schneider JS, Adler CH. The neurobiological basis of cognitive impairment in Parkinson's disease. Mov Disord. 2014;29:634-50. DOI: 10.1002/ mds.25857, PMID: 24757112

3 Braak H, Tredici KD, Rüb U, de Vos RAI, Jansen Steur ENH, Braak E. Staging of brain pathology related to sporadic Parkinson's disease. Neurobiol Aging. 2003;24:197-211. DOI: 10.1016/S0197-4580(02)00065-9, PMID: 12498954

4 Braak H, Rüb U, Gai WP, Del Tredici K. Idiopathic Parkinson's disease: possible routes by which vulnerable neuronal types may be subject to neuroinvasion by an unknown pathogen. J Neural Transm (Vienna). 2003;110:517-36. DOI: 10.1007/s00702-002-0808-2, PMID: 12721813

5 Aarsland D, Zaccai J, Brayne C. A systematic review of prevalence studies of dementia in Parkinson's disease. Mov Disord. 2005;20:1255-63. DOI: 10.1002/mds.20527, PMID: 16041803

6 Aarsland D, Creese B, Politis M, Chaudhuri KR, ffytche DH, Weintraub D, et al. Cognitive decline in Parkinson disease. Nat Rev Neurol. 2017;13:217-31. DOI: 10.1038/ nrneurol.2017.27, PMID: 28257128

7 Hiorth YH, Larsen JP, Lode K, Pedersen KF. Natural history of falls in a population-based cohort of patients with Parkinson's disease: An 8-year prospective study. Parkinsonism Relat Disord. 2014;20:1059-64. DOI: 10.1016/ j.parkreldis.2014.06.023, PMID: 25048614

8 Litvan I, Aarsland D, Adler CH, Goldman JG, Kulisevsky J, Mollenhauer B, et al. MDS task force on mild cognitive impairment in Parkinson's disease: critical review of PD-MCI. Mov Disord. 2011;26:1814-24. DOI: 10.1002/mds.23823, PMID: 21661055

9 Litvan I, Goldman JG, Tröster AI, Schmand BA, Weintraub D, Petersen RC, et al. Diagnostic criteria for mild cognitive impairment in Parkinson's disease: Movement Disorder Society Task Force guidelines. Mov Disord. 2012;27:349-56. DOI: 10.1002/mds.24893, PMID: 22275317

10 Pedersen KF, Larsen JP, Tysnes OB, Alves G. Natural course of mild cognitive impairment in Parkinson disease: A 5-year population-based study. Neurology. 2017;88:767-74. DOI: 10.1212/WNL.0000000000003634, PMID: 28108638

11 Okada K, Kobayashi S, Yamagata S, Takahashi K, Yamaguchi S. Poststroke apathy and regional cerebral blood flow. Stroke. 1997;28:2437-41. DOI: 10.1161/01. STR.28.12.2437, PMID: 9412628

12 Uemura Y, Nomura T, Inoue Y, Yamawaki M, Yasui K, Nakashima K. Validation of the Parkinson's disease sleep scale in Japanese patients: A comparison study using the Pittsburgh Sleep Quality Index, the Epworth Sleepiness Scale and Polysomnography. J Neurol Sci. 2009;287:36-40. DOI: 10.1016/j.jns.2009.09.015, PMID: 19804890

13 Nomura T, Inoue Y, Kagimura T, Kusumi M, Nakashima K. Validity of the Japanese version of the REM Sleep Behavior Disorder (RBD) Screening Questionnaire for detecting probable RBD in the general population. Psychiatry Clin Neurosci. 2015;69:477-82. DOI: 10.1111/pcn.12286, PMID: 25727855
14 Tanaka K, Wada-Isoe K, Nakashita S, Yamamoto M, Nakashima K. Impulsive compulsive behaviors in Japanese Parkinson's disease patients and utility of the Japanese version of the Questionnaire for Impulsive-Compulsive Disorders in Parkinson's disease. J Neurol Sci. 2013;331:76-80. DOI: 10.1016/j.jns.2013.05.013, PMID: 23735774

15 Gibb WR, Lees AJ. The relevance of the Lewy body to the pathogenesis of idiopathic Parkinson's disease. J Neurol Neurosurg Psychiatry. 1988;51:745-52. DOI: 10.1136/jnnp.51.6.745, PMID: 2841426

16 Emre M, Aarsland D, Brown R, Burn DJ, Duyckaerts C, Mizuno Y, et al. Clinical diagnostic criteria for dementia associated with Parkinson's disease. Mov Disord. 2007;22:1689707. DOI: 10.1002/mds.21507, PMID: 17542011

17 Dubois B, Burn D, Goetz C, Aarsland D, Brown RG, Broe GA, et al. Diagnostic procedures for Parkinson's disease dementia: recommendations from the movement disorder society task force. Mov Disord. 2007;22:2314-24. DOI: 10.1002/ mds.21844, PMID: 18098298

18 Dalrymple-Alford JC, MacAskill MR, Nakas CT, Livingston L, Graham C, Crucian GP, et al. The MoCA: well-suited screen for cognitive impairment in Parkinson disease. Neurology. 2010;75:1717-25. DOI: 10.1212/WNL.0b013e3181fc29c9, PMID: 21060094

19 Hobson P, Meara J. Mild cognitive impairment in Parkinson's disease and its progression onto dementia: a 16-year outcome evaluation of the Denbighshire cohort. Int J Geriatr Psychiatry. 2015;30:1048-55. DOI: 10.1002/gps.4261, PMID: 25676160

20 Domellöf ME, Ekman U, Forsgren L, Elgh E. Cognitive function in the early phase of Parkinson's disease, a five-year follow-up. Acta Neurol Scand. 2015;132:79-88. DOI: 10.1111/ ane.12375, PMID: 25644230

21 Boylan LS, Messinis L. Orthostatic hypotension, cognition, and Parkinson disease : Dumbing down by standing up. Neurology. 2017;88:11-2. DOI: 10.1212/WNL.0000000000003468, PMID: 27903812

22 Udow SJ, Robertson AD, MacIntosh BJ, Espay AJ, Rowe JB, Lang AE, et al. 'Under pressure': is there a link between orthostatic hypotension and cognitive impairment in $\alpha$-synucleinopathies? J Neurol Neurosurg Psychiatry. 2016;87:1311-21. DOI: 10.1136/jnnp-2016-314123, PMID: 27613160

23 Martini A, Dal Lago D, Edelstyn NMJ, Grange JA, Tamburin S. Impulse Control Disorder in Parkinson's Disease: A MetaAnalysis of Cognitive, Affective, and Motivational Correlates. Front Neurol. 2018;9:654. DOI: 10.3389/fneur.2018.00654, PMID: 30233478

24 Martini A, Weis L, Fiorenzato E, Schifano R, Cianci V, Antonini A, et al. Impact of Cognitive Profile on Impulse Control Disorders Presence and Severity in Parkinson's Disease. Front Neurol. 2019;10:266. DOI: 10.3389/ fneur.2019.00266, PMID: 30967834 